\title{
BENEFÍCIOS DO MÉTODO CANGURU PARA O ALEITAMENTO MATERNO
}

\section{BENEFITS OF THE KANGURU METHOD FOR BREASTFEEDING}

\section{BENEFICIOS DEL MÉTODO CANGURO PARA LA LACTANCIA MATERNA}

\author{
Roselaine Nascimento da Silva ${ }^{1}$, Fátima Helena Cechetto ${ }^{2}$, Fernando Riegel ${ }^{3}$
}

Como citar esse artigo: Silva RN, Cechetto FH, Riegel F. Benefícios do método canguru para o aleitamento materno. Rev Enferm Atenção Saúde [Internet]. 2021 [acesso em ];10(1):e202110. doi:10.18554/reas.v10i1.4222

\begin{abstract}
RESUMO
Objetivo: verificar os benefícios do Método Canguru para o aleitamento materno. Método: revisão integrativa, por meio das bases de dados SciELO, BDENF e LILACS, os dados foram coletados entre os meses de agosto de 2018 e janeiro de 2019. Para a análise dos dados obtidos, foi utilizada a análise de conteúdo. Resultados: os benefícios do Método Canguru para o aleitamento materno estão relacionados com o fator de promoção, maior adesão e manutenção do aleitamento materno, redução do desmame precoce, melhor sucção, e maior produção de leite. Conclusões: formas indiretas relacionadas ao método Canguru envolvendo o aleitamento materno foram identificadas: o aumento de peso do recém-nascido, redução do tempo de internação do recém-nascido, favorecimento da construção do vínculo mãe-bebê e contribuição para o desenvolvimento global.
\end{abstract}

Descritores: Política Pública; Enfermagem; Método Canguru; Aleitamento Materno.

\begin{abstract}
Objective: To check the benefits that the Kangaroo Method for breastfeeding. Method: Integrative review, through the SciELO, BDENF and LILACS databases, data were collected between August 2018 and May 2019. For the analysis of the obtained data the content analysis was used. Results: The benefits of the Kangaroo Method for breastfeeding found are related to breastfeeding as a promoting factor, greater adherence and maintenance of breastfeeding, reduction of early weaning, presentation of better sucking by baby, and presentation by mothers of higher daily volume in milk production. Conclusions: Indirect forms related to the Kangaroo method involving breastfeeding were identified: increased weight of the baby, reduced length of stay of the newborn, favoring the construction of the mother-baby bond and contribution to global development.
\end{abstract}

Descriptors: Public Policy; Nursing; Kangaroo Method; Breastfeeding.

\footnotetext{
1 Acadêmica de Enfermagem da Faculdade Inedi-Cesuca. Faculdade Inedi-Cesuca, Cachoeirinha-RS. roselainens@gmail.com

${ }^{2}$ Doutora em Ciências da Saúde (ICFUC). Coordenadora do Curso de Enfermagem Faculdade Inedi-Cesuca. Faculdade Inedi-Cesuca, Cachoeirinha-R. Avaliadora do INEP/MEC. fatimaccechetto@ cesuca.edu.br

${ }^{3}$ Doutor em Enfermagem (UFRGS). Mestre em Educação (Unisinos). Professor do Curso de Enfermagem da Universidade Federal de Mato Grosso (UFMT) - Campus Universitário do Araguaia. Faculdade Inedi-Cesuca, Cachoeirinha-RS. fernandoriegel85@gmail.com
} 


\section{RESÚMEN}

Objetivo: Para comprobar los beneficios del Método Canguro para la lactancia materna. Metodo: la revisión integradora, a través de las bases de datos SciELO, BDENF y LILACS, se recopilaron datos entre agosto de 2018 y mayo de 2019.Para el análisis de los datos obtenidos se utilizó el análisis de contenido. Resultados: Los beneficios del método Kangaroo para la lactancia materna encontrados están relacionados con la lactancia materna como un factor promotor, una mayor adherencia y mantenimiento de la lactancia materna, reducción del destete temprano, presentación de una mejor succión por parte de NB y presentación por parte de las madres de mayor volumen diario en la producción de leche. Conclusiones: Se identificaron formas indirectas relacionadas con el método Canguro que involucra la lactancia materna: aumento del peso del bebé, reducción de la duración de la estadía del recién nacido, lo que favorece la construcción del vínculo madre-bebé y la contribución al desarrollo global.

Descriptores: Política Pública; Enfermería; Método Canguro; Lactancia Materna.

\section{INTRODUÇÃO}

O Método Mãe Canguru (MC) foi idealizado e implantado na Colômbia, por volta de 1979 no Instituto Materno-Infantil de Bogotá, pelos doutores Edgar Rey Sanabria e Hector Martinez. No Brasil, o MC foi anunciado em 5 de julho de 2000, pelo Ministério da Saúde, através da Portaria no 693/2000, como uma Norma de Atenção Humanizada do Recém-Nascido de Baixo Peso (RNBP), objetivando a promoção de uma assistência humanizada. ${ }^{1}$

O objetivo principal estava fundamentado na concepção de que o posicionamento do recém-nascido contra o peito da mãe ofertaria maior adequação térmica, substituindo as incubadoras. $\mathrm{O}$ favorecimento da alta hospitalar antecipada, a redução das taxas de infecção hospitalar e a melhoria da qualidade da assistência de saúde, e consequentemente redução do custo para o sistema de saúde estão entre as vantagens do método. ${ }^{1}$
Deste modo, o MC teve sua prática implementada no Brasil como Política Pública Nacional, voltada à atenção perinatal. Com o objetivo de estabelecer uma atenção humanizada e qualificada através do desenvolvimento de intervenção biopsicossocial com que envolve além do cuidado ao (RN) a promoção da participação dos pais e da família. O MC baseia-se no contato pele a pele, iniciado na prática do toque, evoluindo até a posição canguru junto da mãe. Este deve apresentarse apenas vestindo fraldas, em contato pele a pele, junto ao peito dos pais e na posição vertical por um tempo que não deve ser menor do que 15 minutos. Deve ser executada através da assistência de uma equipe de saúde capacitada para garantir segurança e benefícios em sua prática. ${ }^{2}$

Entretanto, no Brasil, o MC possui uma proposta mais ampla que se estende: [...] aos cuidados técnicos com o bebê (manuseio, atenção às necessidades 
individuais, cuidados com luz, som, dor); o acolhimento à família; a promoção do vínculo mãe/bebê e do aleitamento materno; e o acompanhamento ambulatorial após a alta, configurando-se, assim, como estratégia de qualificação do cuidado neonatal. ${ }^{3}$

O MC está sistematizado em três etapas, que envolve o desenvolvimento de ações que acolhem os pais, e viabilizem o comprometimento destes na assistência terapêutica do RN através de sua inserção nos cuidados, oportunizando a construção de vínculos. ${ }^{4}$ A primeira etapa do MC inicia no reconhecimento do trabalho de parto prematuro e peso inferior a 2.500 gramas do $\mathrm{RN}$.

Este método é adotado prioritariamente para bebês com peso menor que 1.500 gramas e inaptos para permanecer no alojamento conjunto, que precisam ficar internados em Unidade de Tratamento Intensivo Neonatal (UTIN). Assim, enquanto o RN permanece na UTIN, a mãe e a família são orientadas com relação ao quadro clínico do bebê e quanto à importância do MC. ${ }^{3}$

É necessário estimular a entrada e a permanência dos pais na UTIN, além da orientação pela equipe de enfermagem sobre as medidas de controle de infecção, estimulando também o toque adequado e o contato pele-a-pele, através da posição Canguru. Esta proposta deve ser sempre que possível e desejada, conforme as condições clínicas do RN. Assim, é preciso ainda promover o estímulo à lactação e a participação dos pais e familiares nos cuidados. $^{5}$

Na segunda etapa do MC, o bebê é mantido continuamente com sua mãe e a posição canguru realizada durante o maior tempo possível, devendo o bebê, para permanecer nessa etapa demonstrar estabilidade clínica, estar em nutrição enteral plena (peito, sonda gástrica ou copo) e obter um peso mínimo de $1.250 \mathrm{~g}$.

Ressalta-se que a mãe deve expressar o seu desejo de participar, ter tempo disponível e uma rede social de apoio, de forma que ocorra um consenso entre mãe, familiares e profissionais da saúde. A mãe também deverá demonstrar habilidade em reconhecer os sinais de estresse e as situações de risco do recémnascido e ter conhecimento e perícia para manejar o bebê em posição canguru. Entretanto, esta etapa também necessita permitir o distanciamento temporário da mãe conforme suas necessidades. $\mathrm{O}$ uso de medicações orais, intramusculares ou endovenosas intermitentes não contra indicam a permanência nessa etapa. ${ }^{6}$

$\mathrm{Na}$ terceira etapa, em que ocorre a alta hospitalar e a manutenção do acompanhamento ambulatorial, o bebê precisa estar com um peso mínimo de $1500 \mathrm{~g}$, em estabilidade clínica e apresentar 
ganho de peso nos três dias que antecedem a alta, recebendo alimentação por aleitamento materno exclusivo. Em casos de necessidade, a complementação da dieta deve ser ofertada por meio de copo ou seringa.

A família deve apresentar segurança com relação ao manejo da criança e ter boa compreensão da importância de mantê-la, também no domicílio, na posição canguru pelo maior tempo possível. Há necessidade de comprometimento com o acompanhamento ambulatorial, em um calendário de três consultas na primeira semana, duas na segunda semana e manutenção de uma consulta da terceira semana em diante, até a aquisição de um peso mínimo de $2500 \mathrm{~g} .^{5}$

Diante deste cenário é importante destacar que o MC pode ser prejudicado devido a várias situações, dentre elas as rotinas neonatais voltadas para o cuidado técnico que afastam a estadia da mãe na unidade neonatal e são obstáculos para amamentação. ${ }^{7}$ Assim, o contato entre mãe e filho no MC permite que as mães continuem produzindo leite, e despertando encorajamento e esperança, propiciados pelo sucesso do aleitamento, e consequentemente, o desejo em cuidar e amamentar o filho. ${ }^{8}$

Dentre os profissionais da equipe multidisciplinar, o enfermeiro pertence à categoria que permanece em tempo integral envolvida com a família e o $\mathrm{RN}$, estabelecendo uma comunicação efetiva e uma linguagem clara, favorecendo o entendimento, o aprendizado e a interação das famílias. Com isso, ressalta-se o propósito e os benefícios do $\mathrm{MC}$, de forma a incentivar a compreensão de sua importância para todos os envolvidos. ${ }^{9}$

Desta forma, é imprescindível que a equipe de enfermagem esteja adequadamente preparada para prestar esta assistência, reconhecendo as vantagens do MC para fomentar suas atividades de incentivo a sua prática. $\mathrm{O}$ objetivo deste estudo foi verificar os benefícios do MC para o aleitamento materno.

\section{MÉTODO}

Trata-se de uma Revisão Integrativa que tem por finalidade reunir e sintetizar achados de estudos realizados, por meio de diferentes metodologias, objetivando contribuir para o aumento do conhecimento relativo ao tema investigado. ${ }^{10}$

A revisão de literatura integrativa utiliza um método sistemático e organizado proposto por Cooper, desenvolvido através de cinco etapas bem definidas. Nestas, são estabelecidos os objetivos do estudo, formulação dos questionamentos que deverão ser respondidos pela pesquisa, por meio da busca de estudos publicados nas bases de dados, através de descritores e critérios de inclusão e exclusão previamente 
determinados. A partir disso, obtém-se a amostra, qualificação e análise dos estudos selecionados, explicitando e expondo os resultados encontrados. ${ }^{11}$ Diante disso, destaca-se a questão norteadora deste estudo: Quais os benefícios que o método canguru oferece para o aleitamento materno?

$\mathrm{Na}$ etapa da coleta de dados foram definidos os critérios de inclusão e exclusão do estudo, bem como a metodologia de busca nas bases de dados. Os critérios de inclusão foram: artigos que respondessem à questão norteadora, redigidos em língua portuguesa, disponíveis na íntegra, resultantes de pesquisas primárias e publicados entre os anos de 2008 a 2018. Como critérios de exclusão, foram descartados manuais, teses, trabalhos de conclusão de curso e dissertações e artigos de revisão.

A pesquisa foi realizada através da busca nas bases de dados: Scientific Electronic Library Online (SciELO), Literatura Latino-Americana e do Caribe em Ciências da Saúde (LILACS), e Base de Dados de Enfermagem (BDENF) disponíveis na plataforma da Biblioteca Virtual em Saúde (BVS). Os descritores de busca foram: Política Pública, Enfermagem, Método Canguru, Aleitamento Materno, utilizando o operador booleano “AND". A coleta de dados ocorreu no período de março a maio de 2019.
Foram encontrados 887 artigos, sendo 154 artigos na LILACS, 228 artigos na Scielo e 505 artigos na BDENF. Ao proceder a seleção a partir da aplicação dos critérios de inclusão foram préselecionados 148 artigos e 855 foram excluídos por não responder à questão norteadora ou por duplicidade. Após leitura dos títulos e resumos foram selecionados 52 artigos, e desses, apenas 18 artigos relacionaram-se ao objetivo deste estudo. Foram respeitados os direitos autorais de acordo com a Lei de Direitos Autorais $\mathrm{n}^{\circ}$ 12.853 de 14 de agosto de $2013 .^{12}$

\section{RESULTADOS E DISCUSSÃO}

Os achados desta revisão integrativa evidenciaram os benefícios do MC para o AM e foram organizados em categorias de análise que envolvem os seguintes tópicos: a) relacionados diretamente com $\mathrm{o}$ aleitamento materno. O MC mostrou-se fator de promoção, maior adesão e manutenção do aleitamento materno, redução do desmame precoce, apresentação de melhor sucção pelos RN, e mães apresentando maior volume diário na produção de leite; b) de formas indiretas relacionadas ao $\mathrm{MC}$ envolvendo o $\mathrm{AM}$ foram identificados o aumento de peso do $\mathrm{RN}$, redução do tempo de internação do $\mathrm{RN}$, favorecimento da construção do vínculo mãe-bebê e contribuição para o desenvolvimento global do bebê. 
$\mathrm{O} \mathrm{MC}$ revelou-se uma estratégia de grande importância para a promoção, adesão e manutenção do aleitamento materno, identificados em todos os estudos avaliados (A1 a A18), e incentivador do AM de forma exclusiva, apontado por sete estudos (A1, A2, A4, A5, A10, A13 e A16).

Foi evidenciado que o MC favorece maior frequência, periodicidade e duração no aleitamento materno com livre demanda, uma vez que consiste em fonte de nutrição e proteção ao bebê nos seis primeiros meses de vida. $\mathrm{O}$ MC contribui com fatores positivos importantes para o futuro do RN. Um de seus objetivos é incentivar o AM; para tanto, a mãe necessita ser adequadamente orientada com relação aos benefícios do aleitamento e os cuidados na amamentação. ${ }^{13}$

O Método Canguru é apontado como facilitador da amamentação materna, pois oportuniza o contato pele a pele e a permanência da mãe junto ao seu filho prematuro, e pais e familiares têm manifestado satisfação e interesse pelo MC frente à recuperação do bebê. ${ }^{14}$

O longo período de internação do RN de baixo peso, o uso prolongado de sonda gástrica e a privação de estímulos sensoriais na região oral, retardam a transição da dieta de gavagem para via oral e a maturação motora oral, retardando também a alta hospitalar. Desta forma, o contato precoce e a execução da técnica sonda-peito favorecidos no método canguru, apresentaram redução do tempo de transição das etapas de alimentação somente por sonda ou sua complementação. ${ }^{15}$

$\mathrm{O}$ MC possui inúmeros benefícios para a puérpera e para o bebê, permitindo uma vivência única, na qual a mãe se encontra próxima de seu filho semelhante ao momento intraútero. Esse contato facilita o aleitamento materno, evidenciado através dos relatos das puérperas, pois possibilita maior facilidade ao bebê para alcançar o seio pela posição proporcionada pelo método, proximidade que o torna efetivo. Assim, essa vivência proporciona às mães o sentimento de inserção integral na recuperação do seu bebê, aumentando consequentemente o vínculo. ${ }^{1}$

O MC como estratégia para evitar o desmame precoce foi apontado por três estudos (A3, A5 e A15), uma vez que, apontam fatores que promovem a interrupção do aleitamento, tais como: desestímulos, campanhas comerciais equivocadas e alterações do papel da mulher na sociedade, permitindo que crenças e mitos ainda influenciem o comportamento em relação à amamentação. Além disso, os grupos sociais nos quais a mulher está inserida, especialmente o grupo familiar possui grande influência na prática do AM, sendo a família a principal transmissora de crenças e valores. ${ }^{16}$ 
O retorno da mãe ao trabalho, desinteresse da mãe em amamentar, mitos e preconcepções sobre o aleitamento, problemas mamários e falta de orientação no pré-natal são apontados como fatores de risco para o desmame precoce. Do núcleo familiar, as avós possuem maior influência sobre a mãe quanto à amamentação. $\mathrm{O}$ desmame ocorre pela concepção de que seu leite é insuficiente, devido a problemas mamários, à falta de tempo e apoio para amamentar, além da sensação de sobrecarga e frustração. ${ }^{17} \mathrm{O}$ benefício do $\mathrm{MC}$ para $\mathrm{o}$ aumento da produção de leite pela mãe e no desenvolvimento da sucção correta e efetiva pelo $\mathrm{RN}$ ficaram evidentes em dois estudos (A15, A17).

Fatores como a crença infundada de que "o leite materno é fraco", bem como a ideia de pouca produção de leite influenciam negativamente na manutenção do AM. Esses mitos são desfeitos quando a mãe recebe atenção na prática do $\mathrm{MC}$ e está envolvida no cuidado do seu filho desde o nascimento. Quando ela é adequadamente orientada pela equipe de saúde sobre a composição do leite materno os resultados da amamentação tornam-se positivos.

Faz-se importante destacar no ato da orientação que a produção de leite é estimulada pelas mamadas, ou seja, quanto mais o bebê sugar, mais leite será produzido; também faz-se importante destacar que não há necessidade de introdução de outros alimentos como complementos antes dos seis meses, e que o uso de mamadeiras e/ou chupetas interferem na produção do leite e no AM. ${ }^{16}$

O método como abordagem de cuidado proporciona o contato da mãe/família por longos períodos com a equipe de saúde. Com isso, é possível afirmar que este também é um dos benefícios do método para a promoção do AM e sua manutenção após a alta. O MC evita o desmame precoce, visto que a enfermagem conta com maior tempo para orientar e auxiliar adequadamente a família.

Esta revisão integrativa também evidenciou formas indiretas de benefícios relacionados ao Método Canguru envolvendo o Aleitamento Materno. Um dos benefícios do MC apontado por quatro estudos (A1, A2, A7, A8) está relacionado ao aumento de peso do RN. Com relação ao ganho de peso diário, o MC reduz o tempo para ganho de peso quando comparado ao método convencional, pois facilita a amamentação, contribuindo positivamente para o ganho ponderal de peso. ${ }^{18}$

O MC através do contato pele a pele promove a termorregulação, a estabilidade dos sinais vitais e o aleitamento materno, produzindo a homeostase do $\mathrm{RN}$, condição de importância maior para o aumento de peso. ${ }^{19} \mathrm{~A}$ redução do tempo de internação do $\mathrm{RN}$ foi descrito por três estudos (A1, A3, A17). Diante dos benefícios apresentados 
pelo MC ocorre a redução do tempo de internação na UTI Neonatal, possibilitando a alta hospitalar com maior brevidade, pois o $\mathrm{RN}$ se torna mais rapidamente apto a ir para a residência com sua família. ${ }^{20}$

Estudos evidenciaram as contribuições o MC no estabelecimento do vínculo bebê-família, estimulando a participação dos pais no cuidado de seu filho, de forma que possam compreender todo o processo da prematuridade. Com isso, busca-se o preparo para a realização dos cuidados domiciliares, o que contribuirá para a alta hospitalar com maior brevidade e segurança. ${ }^{21}$

O favorecimento da construção do vínculo mãe-bebê pelo MC foi pontuado em seis estudos (A6, A7, A14, A16, A17, A18). $\mathrm{O}$ período que mãe e bebê permanecem juntos após o nascimento desencadeia uma sequência de eventos sensoriais, hormonais, fisiológicos, imunológicos e comportamentais que favorecem a formação do vínculo entre o binômio mãebebê. Nos estudos, as mães relataram que através do MC é viabilizada a manutenção da posição canguru que ultrapassa o ato de tocar e o contato pele a pele, proporcionando que as mães vivenciem sentimentos de emoção e alegria, uma vez que se sentiram mais próximas de seus filhos pegando-os no colo, transmitindo carinho e calor, fortalecendo o apego, e uma forma de sentir-se unidos, como um só novamente. ${ }^{22}$

Frente ao exposto, o MC promove o exercício maternal, de forma que a puérpera vivencia experiências sensoriais diretamente com o filho, potencializando seu papel enquanto cuidadora, impactando sobre os sentimentos negativos experimentado nos primeiros dias de internação, superando-os de forma a estabelecer o apego e o vínculo com seu filho. ${ }^{21,24}$

A contribuição do MC para o desenvolvimento global do $\mathrm{RN}$ foi apontada por três estudos (A10, A14, A18). O MC é importante também para o crescimento do $\mathrm{RN}$ de baixo peso, pois auxilia no aumento de peso, na redução dos índices da bilirrubina não conjugada sanguínea evitando a icterícia neonatal, aumentando os níveis adequados de glicose no sangue, bem como promovendo o desenvolvimento neurológico, intelectual, psicológico e biológico, contribuindo no desenvolvimento da coordenação, deglutição e sucção, e da fala. ${ }^{13}$

\section{CONSIDERAÇÕES FINAIS}

Os benefícios do MC evidenciados com este estudo impactam positivamente na qualidade do aleitamento materno. O MC estimula a promoção do AM, pois coloca a mãe em contato direto com o bebê no momento em que o RN encontra-se estável 
e em condições de fazê-lo, aumentando a adesão das mães e o seguimento da prática do AM após a alta hospitalar, reduzindo a incidência do desmame precoce, uma vez que durante a prática do MC as mães são orientadas pelos profissionais de enfermagem quanto à amamentação exclusiva.

Os RNs têm demonstrado melhor processo de sucção e deglutição quando inseridos no $\mathrm{MC}$, resultando em melhores práticas de AM, e as mães, frente ao sucesso da pega do RN no seio, mantém um maior volume diário de produção de leite. Com relação às formas indiretas relacionadas ao MC envolvendo o AM foram identificados: o aumento de peso do $\mathrm{RN}$ gerado pelo sucesso do aleitamento, a redução do tempo de internação do $\mathrm{RN}$ também relacionado à estabilidade e o ganho de peso. Outros benefícios envolvem o favorecimento da construção do vínculo mãe-bebê pelo estímulo do contato pele a pele e a contribuição para o desenvolvimento físico, psicológico e social da criança.

O MC apresenta inúmeros benefícios para o recém-nascido, para a mãe e toda a família, mas o fator benéfico mais evidenciado está relacionado com a promoção do aleitamento materno. Deste modo, é de extrema importância que os profissionais de enfermagem estejam adequadamente preparados para assistir mãe e bebê durante a internação e na promoção do MC, aproveitando este espaço para conscientização e incentivo ao aleitamento materno.

\section{LIMITAÇÕES DO ESTUDO}

Este estudo tem como limitações a inclusão de artigos exclusivamente no idioma português, que nos leva a resultados parciais na revisão integrativa. Sugerem-se a realização de outros estudos sobre a temática com publicações em outros idiomas. Desta forma, possibilita-se um resultado mais fidedigno da avaliação do MC e sua relação com o aleitamento materno, enfatiza-se a influência dos aspectos culturais nos resultados obtidos com este método.

\section{REFERÊNCIAS}

1. Neves PN, Ravelli APX, Lemos JRD. Atenção humanizada ao recém-nascido de baixo-peso (método Mãe Canguru): percepções de puérperas. Rev Gaúch Enferm. [Internet]. mar 2010 [citado em 23 jul 2020]; 31(1): 48-54. Disponível em: http://www.scielo.br/scielo.php?script=sci _arttext\&pid=S198314472010000100007\&lng=en. doi:10.159 0/S1983-14472010000100007

2. Ministério da Saúde (Brasil), Secretaria de Atenção à Saúde, Departamento de Ações Programáticas Estratégicas. Atenção humanizada ao recém-nascido: método canguru: manual técnico. 3ed. Brasília, DF: Ministério da Saúde; 2017.

3. Gontijo TL, Meireles AL, Malta DC, Proietti FA, Xavier CC. Avaliação da implantação do cuidado humanizado aos recém-nascidos com baixo peso: método canguru. J Pediatr (Rio J.) [Internet]. fev 2010 [citado em 23 jul 2020]; 86(1):33-39. 
Disponível em:

http://www.scielo.br/scielo.php?script=sci

_arttext\&pid=S0021-

$75572010000100007 \& \operatorname{lng}=$ en. doi:10.1590/S0021-75572010000100007

4. Stelmak A, Freire M. Aplicabilidade das ações preconizadas pelo método canguru.

Rev Pesqui. (Univ Fed Estado Rio J, Online) [Internet]. jul 2017[citado em 23 jul 2020]; 9(3):795-802. Disponível em: http://seer.unirio.br/cuidadofundamental/ar ticle/view/4429/pdf_1

5. Borck M, Santos EKA. Terceira etapa método canguru: convergência de práticas investigativas e cuidado com famílias em atendimento ambulatorial. Rev Gaúch Enferm. [Internet]. dez 2010 [citado em 23 jul 2020]; 31(4):761-68. Disponível em: http://www.scielo.br/scielo.php?script=sci _arttext\&pid=S1983-

14472010000400021\&lng=pt. doi: 10.1590 /S1983-14472010000400021

6. Ministério da Saúde (Brasil). Portaria $n^{\circ}$ 1.683, de 12 de julho de 2007. Aprova, na forma do Anexo, a Normas de Orientação para a Implantação do Método Canguru [internet]. Brasília, DF: Ministério da Saúde; 2007 [citado em 30 set 2018]. Disponível em: http://bvsms.saude.gov.br/bvs/saudelegis/g m/2007/prt1683_12_07_2007.html.

7. Almeida H, Venancio SI, Sanches MT

C, Onuki D. Impacto do método canguru nas taxas de aleitamento materno exclusivo em recém-nascidos de baixo peso. $\mathrm{J}$

Pediatr (Rio J.) [Internet]. jun 2010

[citado em 23 jul 2020]; 86(3):250-53.

Disponível em:

http://www.scielo.br/scielo.php?script=sci _arttext\&pid=S0021-

$75572010000300015 \& \operatorname{lng}=$ en. doi: 10.159 0/S0021-75572010000300015

8. Rolim KMC, Vidal AF, Mariano MA, Campos ACS, Frota. MA. Percepção das mães sobre aleitamento em prematuros da unidade canguru de uma maternidade de Fortaleza - CE. Rev Rene. [Internet]. abr/jun 2008 [citado em 23 jun 2020]; 9(2):54-63. Disponível em: http://www.periodicos.ufc.br/rene/article/v iew/4998/3681

9.Coêlho LS, Dias AA, Landim CAP, Lima JVF, Silva MNP, Abdalla CM. Amamentação do recém-nascido prematuro no método canguru: percepções de puérperas sobre o cuidado de enfermagem. Rev Pesqui. (Univ Fed Estado Rio J, Online) [Internet]. dez 2013 [citado em 04 set 2018]; 5(6):284-92.

Disponível em:

https://www.redalyc.org/articulo.oa?id=50 5750944031

10. Ribeiro OMPL, Martins MMFPS, Tronchin DMR. Modelos de prática profissional de enfermagem: revisão integrativa da literatura. Rev Enf Ref. [Internet]. set 2016 [citado em 23 jul 2020]; serIV(10):125-33. Disponível em: http://www.scielo.mec.pt/scielo.php?script =sci_arttext\&pid=S0874-

02832016000300014\&lng=pt. doi: 10.127 07/RIV16008

11.Cooper HM. Scientific guidelines for conducting integrative research reviews. Rev Educ Res. 1982; 52(2):291-302. 12. Presidência da República (Brasil). Lei $\mathrm{n}^{\circ}$ 9.610, de 19 de fevereiro de 1998. Altera, atualiza e consolida a legislação sobre direitos autorais [Internet]. D.O.U., Brasília, DF, 20 fev 1998 [citado em 23 jun 2020]. Disponível em: http://www.planalto.gov.br/ccivil_03/leis/l 9610.htm

13.Marques CRG, Neris ILF, Carvalho MVA, Menezes MO, Ferrari YAC.

Metodologia canguru: benefícios para o recém-nascido pré-termo. Caderno de Graduação: ciências biológicas e de saúde [Internet]. 2016 [citado em 23 jun 2020]; 3(3):65-78. Disponível em:

https://periodicos.set.edu.br/cadernobiologi cas/article/view/3134/1988

14. Silva A, Alencar-Salmeron N, CocaLeventhal L. Percepção dos pais em relação ao "Método Mãe-Canguru". Saúde Colet. [Internet]. 2012 [citado em 23 jun 2020]; 56(9):46-50. Disponível em: https://www.redalyc.org/articulo.oa?id=84 223413003 
15. Medeiros AMC, Ramos BKB, Bomfim DLSS, Alvelos CL, Silva TC, Barreto IDC et al. Tempo de transição alimentar na técnica sonda-peito em recém-nascidos baixo peso do método canguru.

CoDAS [Internet]. 2018 [citado em 23 jul 2020]; 30(2):e20170092. Disponível em:

http://www.scielo.br/scielo.php?script=sci _arttext\&pid=S2317-

17822018000200306\&lng=en

16. Barbosa JAG, Santos FPC, Silva PMC.

Fatores associados à baixa adesão ao

aleitamento materno exclusivo e ao

desmame precoce. Rev Tecer. [Internet].

2013 [citado em 23 jun 2020]; 6(11):154-

65. Disponível em:

https://www.metodista.br/revistas/revistasizabela/index.php/tec/article/view/352/408

17.Abreu FCP, Fabbro MRC, Wernet M.

Fatores que intervêm na amamentação exclusiva: revisão integrativa. Rev Rene.

[Internet]. 2013 [citado em 23 jun

2020];14(3):610-19. Disponível em:

https://www.redalyc.org/pdf/3240/3240279

91017.pdf

18.Santana JCB, Assis APO, Silva CCD,

Quites HFO. Método mãe canguru e suas implicações na assistência: percepção da equipe de enfermagem. Enferm Rev. [Internet]. 2013 [citado em 23 jun 2020]; 16(1):34-48. Disponível em:

http://periodicos.pucminas.br/index.php/en fermagemrevista/article/view/13017 19. Casati OS, Oliveira CS, Paula S.

Método mãe canguru e suas associações nos benefícios dos recém-nascidos de baixo peso. Uniciências [Internet]. 2010 [citado em 23 jun 2020];14(1):135-46. Disponível em:

file:///C:/Users/S\%C3\%94NIA/Downloads 1858.pdf

20. Silva LJ, Leite JL, Silva TP, Silva IR, Mourão PP, Gomes TM. Desafios gerenciais para as melhores práticas do Método Canguru na UTI Neonatal. Rev Bras Enferm. [Internet]. 2018 [citado em 23 jun 2020]; 71(Supl 6):2783-91.

Disponível em: https://www.scielo.br/j/reben/a/tw7vv6nxJ vX4kQr6J6JCdSP/abstract/?lang=en
21.Santos MH, Azevedo Filho M.

Benefícios do método mãe canguru em recém-nascidos pré-termo ou baixo peso: uma revisão da literatura. Univ, Ciênc Saúde [Internet]. 2016 [citado em 23 jun 2020]; 14(1):67-76. Disponível em: https://www.publicacoesacademicas.unice ub.br/cienciasaude/article/view/3477/3071 22. Zirpoli DB, Mendes RB, Barreiro MSC, Reis TS, Menezes AF. Método beneficios canguro: una revisión integradora. Rev Pesqui. (Univ. Fed. Estado Rio J., Online) [Internet]. 2019 [citado em 21 jul 2020]; 11(2):547-54. Disponível em: https://ciberindex.com/index.php/ps/about

RECEBIDO: 31/12/2019

APROVADO: $17 / 12 / 2020$

PUBLICADO: 06/2021 